\title{
Parcours atypique d'une chercheure engagée : à la croisée des mouvements sociaux et de la géographie sociale
}

Entretien avec Anne-Latendresse

Anne Latendresse, Anne-Laure Pailloux and Fabrice Ripoll

\section{OpenEdition}

Journals

\section{Electronic version}

URL: http://journals.openedition.org/cdg/5145

DOI: $10.4000 /$ cdg. 5145

ISSN: 2107-7266

Publisher

UMR 245 - CESSMA

Electronic reference

Anne Latendresse, Anne-Laure Pailloux and Fabrice Ripoll, "Parcours atypique d'une chercheure

engagée : à la croisée des mouvements sociaux et de la géographie sociale", Carnets de géographes [Online], 12 | 2019, Online since 17 March 2021, connection on 17 March 2021. URL: http:// journals.openedition.org/cdg/5145 ; DOI: https://doi.org/10.4000/cdg.5145

This text was automatically generated on 17 March 2021.

\section{(c) (†) $\ominus$}

La revue Carnets de géographes est mise à disposition selon les termes de la Licence Creative Commons Attribution - Pas d'Utilisation Commerciale - Pas de Modification 4.0 International. 


\title{
Parcours atypique d'une chercheure engagée : à la croisée des mouvements sociaux et de la géographie sociale
}

\author{
Entretien avec Anne-Latendresse
}

Anne Latendresse, Anne-Laure Pailloux and Fabrice Ripoll

1 En novembre 2018, nous avons rencontré Anne Latendresse, professeure de géographie sociale et urbaine à l'Université du Québec à Montréal (UQAM), afin d'échanger sur son parcours et ses travaux. Il se trouve en effet qu'elle fait partie des rares géographes à avoir travaillé dès les années 1990 sur la dimension spatiale des mouvements sociaux, et plus encore à l'avoir fait dans le cadre d'une thèse de doctorat, intitulée : Processus de déstructuration-restructuration et dynamique palestinienne à Jérusalem-Est depuis 1967. En outre ses travaux sont sans doute encore méconnus en France. Pour toutes ces raisons, il nous a paru intéressant de publier un entretien dans ce dossier.

\section{Une improbable entrée en thèse}

2 Anne-Laure Pailloux \& Fabrice Ripoll : Travailler en thèse sur des questions qui croisent la géographie et l'objet mouvements sociaux, a fortiori dès les années 1990, ça semble quelque chose d'assez nouveau et improbable. Est-ce que tu pourrais nous raconter un peu ton parcours et comment tu en es arrivée à cette orientation de recherche?

3 Anne Latendresse: Je dirais qu'il y a au moins deux facteurs pour expliquer mon parcours académique, et la façon dont j'ai orienté ma vie professionnelle, les deux étant liés. Le premier réside dans mes origines familiale et ouvrière. Le second facteur renvoie à l'expérience syndicale de mon père qui, en tant que travailleur, a pris part à des grèves et à des actions collectives afin d'améliorer leurs conditions de travail. Grandir dans un milieu ouvrier a certainement influencé mon parcours, et mon engagement au sein du mouvement étudiant, a sans doute influencé ma volonté de 
m'engager au sein de mouvements de solidarité internationale. Ces deux facteurs sont distincts mais dans les faits, ils convergent, et permettent, en partie du moins, d'expliquer le choix de ma recherche doctorale et mes intérêts de recherche. Ma thèse porte sur le conflit israélo-palestinien, et mes travaux de recherche se concentrent surtout sur les mouvements sociaux urbains, les luttes féministes et celles menées pour le logement, et le droit à la ville.

Le premier facteur comme je viens de le mentionner, réside dans mes origines familiale et ouvrière. Mon père, qui n'a pas eu la chance d'étudier, a travaillé toute sa vie comme soudeur. D'abord, dans une usine qui produit de l'aluminium dans la région du Saguenay. À la suite d'une mise à pied, il quitte la région laissant derrière sa famille pour aller travailler dans un chantier maritime, situé à Lauzon ${ }^{1}$ une petite ville située sur la rive-sud de Québec. Un an plus tard, ma mère et les trois enfants le suivent. Notre vie n'est pas facile, celle de mon père est ponctuée de périodes de mise à pied, de chômage et de grèves, sans compter les heures de travail supplémentaires et les 12 années où il travaille de nuit pour augmenter ses revenus. Nous ne vivions pas dans la pauvreté, mais dans une certaine précarité. En côtoyant d'autres enfants et leurs parents mieux nantis, je saisis rapidement la différence entre le « eux » et le « nous ». Le « eux », dans ce cas-ci les enfants de classe moyenne ou de la petite bourgeoisie dont les parents possèdent une maison, une ou deux voitures, une résidence secondaire, et inscrivent leurs enfants à l'école privée, et dans les clubs sportifs ou de musique. Le «nous » auquel j'appartiens, constitué d'enfants de milieux ouvriers qui habitent dans les quartiers populaires à proximité du chantier maritime. Ma famille, locataire, vit dans un modeste logement dont les fenêtres, mal isolées, givrent l'hiver. Ce qu'il faut retenir de ce long préambule, c'est que les origines modestes de ma famille et le quartier où nous habitions m'ont permis de saisir les enjeux liés aux inégalités. Bref, on peut dire qu'assez jeune, j'ai compris ce que les géographes désignent comme «l'effet de classe » et «l'effet de lieu » (Frémont et al., 1984), et cela m'a rendue très sensible aux injustices et aux inégalités sociospatiales.

5 J'ajouterais à cela, et c'est le deuxième facteur, le fait que mon père a pris part à de longues grèves qui ont permis aux ouvriers d'obtenir des gains en termes de conditions de travail, de salaire, et de mesures de santé et sécurité au travail. Ces grèves m'ont permis de comprendre que les actions collectives, pour reprendre la terminologie propre aux théories sur les mouvements sociaux, pouvaient, potentiellement du moins, mener à des avancées significatives pour les familles, et parfois même à des transformations pour l'ensemble de la société. Le Québec de cette époque vivait une "révolution tranquille» à laquelle le mouvement syndical a grandement contribué. Toute jeune, j'ai compris que les luttes n'étaient ni données, ni gagnées à l'avance, et que ce sont les femmes et les hommes qui par leurs actions peuvent transformer les rapports de pouvoir. Vers la fin de mon adolescence, je prends davantage conscience des inégalités entre hommes et femmes, ce qui explique en grande partie mon engagement de chercheure pour les enjeux et luttes portés par les mouvements féministes ici et ailleurs dans le monde.

6 Cette sensibilité aux inégalités et aux injustices, qui m'habite encore aujourd'hui, se traduit donc par un intérêt pour les mouvements sociaux et la solidarité internationale. Vers la fin des années 1970, lors de mes études collégiales ${ }^{2}$, les peuples nicaraguayen et salvadorien mènent respectivement des luttes de libération nationale. En lien avec l'actualité internationale, des enseignant.e.s nous ont introduisent à l'histoire de 
l'Amérique latine, marquée par la colonisation, mais aussi par l'existence de mouvements populaires pour s'affirmer face à l'impérialisme états-unien, et contre les dictateurs en place qui s'en font les complices. À l'université, cet intérêt pour les pays du Sud global va m'inciter à m'engager activement dans des réseaux de solidarité internationale avec l'Afrique du Sud à l'époque de l'apartheid, et avec le peuple palestinien dont je découvre l'histoire grandement marquée par le projet sioniste et la colonisation, la tragédie de la $\mathrm{Nakba}^{3}$ et la dépossession territoriale par Israël.

7 Après avoir terminé un baccalauréat en communications et un certificat en études arabes, j'interromps mes études pour travailler dans une ONG de solidarité internationale, soit le Centre d'études arabes pour le développement (CEAD), de 1987 à 1991, et je m'implique activement dans une coalition pour la paix et la justice en Palestine.

8 ALP \& FR : Donc tu as arrêté des études pour travailler dans une ONG, puis tu fais un retour aux études pour faire une thèse sur des mouvements sociaux avec un angle géographique. Peux-tu nous en dire quelques mots?

9 AL : En effet, cet emploi au CEAD a été important dans mon parcours, il m'a permis de développer une bonne compréhension des enjeux de développement dans diverses régions du monde arabe, de côtoyer des intellectuel.le.s de cette région du monde, d'assister ou d'organiser des conférences, des séminaires au Québec ou dans le monde arabe. J'ai aussi appris à connaître de l'intérieur le milieu des $\mathrm{ONG}$, et je me suis familiarisée avec les institutions internationales, etc. Cependant mes tâches étaient souvent de nature organisationnelle et logistique. Or, je voulais développer une certaine expertise sur la région, ce qui nécessitait de poursuivre des études supérieures. J'hésitais alors entre une maîtrise en science politique à l'UQAM où exerçait Thierry Hentsch ${ }^{4}$, un excellent professeur qui travaillait sur l'Orient et qui était fin connaisseur du conflit israélo-palestinien, et des études au département de géographie où pourtant aucun professeur n'avait d'expertise sur le monde arabe ou sur le Machrek... Étonnamment, j'ai opté pour le pari le plus risqué ! En fait, j'avais constaté l'abondance de travaux sur le conflit israélo-palestinien en science politique, et je voyais difficilement ce que je pourrais apporter d'original sur le plan scientifique. Parallèlement, j'ai fait quelques séjours au Machrek qui m'ont permis de réaliser que presque tous les conflits majeurs dans cette région du monde étaient liés à des enjeux territoriaux, soit autour de frontières disputées, de zones habitées par certains groupes ethnoculturels comme les Kurdes, ou encore autour de secteurs géographiques dotés de ressources comme l'eau ou le pétrole. Mes séjours en Cisjordanie et dans la bande de Gaza occupés par Israël, alors que je travaillais pour le CEAD, m'avaient permis de voir de mes propres yeux la dépossession territoriale du peuple palestinien. J'y ai observé la confiscation des terres appartenant à des familles palestiniennes pour y construire des colonies de peuplement israéliennes érigées illégalement du point de vue du droit international. Par ailleurs, à cette époque, il y avait très peu d'études qui abordaient le conflit israélo-palestinien dans une perspective géographique, ce qui m'étonne encore aujourd'hui puisqu'à mon avis, le territoire constitue le cœur de ce conflit. J'ai donc opté pour le programme de maîtrise en géographie. Avant-même que j'aie terminé ma maîtrise, l'UQAM ouvrait un programme de doctorat en études urbaines. Un professeur de géographie pour qui j'avais beaucoup de respect, m'a encouragée à postuler. Moi qui suis issue d'un milieu ouvrier, l'idée de la maîtrise ne m'était jamais passée par la tête, imaginez des études doctorales ! J'ai réfléchi à cette proposition, j'en ai parlé autour de 
moi. Mon complice et compagnon de vie de l'époque terminait son doctorat, et il m'a encouragée à faire de même. J'ai donc décidé d'aller de l'avant, ce que je n'ai jamais regretté.

10 Comme on le voit, il arrive que des parcours académique ou professionnel reposent sur des opportunités qui s'offrent à nous, plutôt que sur une trajectoire bien précise et tracée à l'avance.

\section{La conception du sujet de thèse}

11 ALP \& FR : Mais comment arrives-tu à cette conviction que les enjeux géopolitiques et socio-spatiaux comme tu dis sont primordiaux, et à cette problématique de thèse ?

12 AL : Euh...j'ai un parcours un peu éclectique. J'ai omis un petit épisode qui va être déterminant pour mon sujet de thèse. En 1991, je fais un court séjour en Afrique du Sud alors que le Congrès national Sud-africain (ANC) tenait son premier congrès légal à l'intérieur du pays 5 . Ce pays entre alors dans une ère de transition démocratique à la suite d'un soulèvement populaire qui a lieu de 1984 à 1986. Cette période de turbulences est marquée par une diversité d'actions collectives et de grèves dans divers secteurs d'activités qui paralysent presque complètement le pays. Déployées par des jeunes, des étudiant.e.s, des travailleur.euse.s et des femmes, ces actions sont souvent appelées par l'ANC, et s'inscrivent dans un vaste mouvement social qui, appuyé par un réseau de solidarité internationale, va finalement forcer le gouvernement en place à abolir l'apartheid, à organiser des élections libres et démocratiques, et à s'engager dans un processus de réformes.

13 Une délégation du Québec, à laquelle je participe, se rend en Afrique du Sud en 1991 pour assister à cet événement historique. Comme il s'agit de la tenue des premières élections libres et démocratiques de l'ANC, des comités sud-africains organisent différentes activités pour les observateur.trice.s et sympathisant.e.s dont une tournée dans les townships, des quasi bidonvilles établis aux abords des plus grandes villes blanches et réservés à la population noire qui constitue la majeure partie de la main d'œuvre dans les mines, l'agriculture et les industries (détenues par la bourgeoisie blanche). Comme les Noir.e.s n'ont pas le droit de dormir dans les villes blanches, ils et elles franchissent de longues distances entre les villes blanches et les townships, pour travailler. Leurs conditions de vie sont misérables. D'ailleurs, entre 1984 et 1986, les townships ont été des lieux de mobilisations populaires où les habitant.e.s dénoncent la quasi absence de services municipaux et boycottent les taxes municipales, de même qu'ils revendiquent de meilleurs logements. Ces luttes autour d'enjeux urbains étaient bien sûr liées aux mobilisations contre l'apartheid qui traversent le pays, et qui sont appuyées à l'international.

Pendant notre mission là-bas, nous rencontrons des militant.e.s et professeur.e.s d'universités, de même que certain.e.s leaders d'organisations sud-africaines. Parmi eux, il y a un groupe spécialisé en aménagement et planification du territoire qui nous explique la ségrégation spatiale ou si vous voulez l'organisation ethno-spatiale du territoire national. Je comprends tout à coup comment la planification et l'aménagement peuvent être au service d'un programme politique ou idéologique, dans ce cas-ci celui de l'État sud-africain qui lie capitalisme, colonialisme et racisme. 
15 J'ajoute un dernier élément à propos de ce séjour en Afrique du Sud qui, bien que banal en apparence, influence ma recherche doctorale. Lors d'une visite à l'Université Kwazulu-Natal, je fais un petit tour à la librairie où je tombe, un peu par hasard, sur le livre The City and the Grassroots de Manuel Castells publié en 1983. Je l'achète et me plonge dans la thèse de ce sociologue des mouvements sociaux qui avait étudié avec Alain Touraine. Dans cet ouvrage, Castells, après avoir analysé des luttes urbaines menées dans des villes de différents pays à différentes époques de l'histoire, démontre que « dans de rares exceptions dans l'histoire », des actions collectives menées dans des villes autour d'enjeux urbains peuvent contribuer à des transformations sociales historiques. Pour développer cette hypothèse, il met de l'avant le cas des comités de citoyens de Madrid qui, tout en se mobilisant sur des enjeux locaux, ont contribué à la chute du régime dictatorial de Franco. La lecture de cet ouvrage prend tout son sens pour moi alors que je me trouvais en Afrique du Sud. Cela me permet de comprendre que des actions collectives se produisant autour d'enjeux locaux ou "urbains ${ }^{6}$ " peuvent s'arrimer à un mouvement social plus vaste dont la portée transformatrice va bien au-delà de l'échelle locale.

À mon retour d'Afrique du Sud, je m'inspire de l'approche mise de l'avant par Castells pour développer un cadre théorique et une grille d'analyse qui lient les actions collectives menées aux échelles locale et nationale. Un peu comme cela a été le cas en Afrique du Sud, l'Intifada ${ }^{7}$, un vaste mouvement palestinien de résistance populaire se déploie à partir de la fin de 1987 dans les villes, les villages et les camps de réfugiés qui s'organisent localement, suivant ainsi les appels lancés par un leadership palestinien établi dans les territoires occupés militairement par Israël. Dans les principaux secteurs d'activités comme la santé, l'enseignement, le commerce, les hommes et les femmes s'organisent afin de prendre part à la résistance. Les jeunes approvisionnent les populations des villages ou des camps de réfugié.e.s soumis à des couvre-feu par l'armée israélienne, des enseignant.e.s mettent sur pied des comités d'éducation populaire visant à prendre en charge l'éducation des enfants alors que l'armée israélienne ferme les écoles. Des comités de femmes et des petites coopératives agricoles transforment leurs fruits et légumes pour les vendre ou les distribuer aux familles dans le besoin. Bref, un mouvement de résistance pacifique et populaire s'organise à l'échelle locale, partout sur l'ensemble du territoire de la Cisjordanie et de la bande de Gaza, de même qu'à Jérusalem-Est. Comme il se déploie dans les territoires occupés par Israël, un leadership palestinien clandestin émerge afin de coordonner ces actions collectives et d'établir une stratégie. Et il entretient des liens étroits avec l'O.L.P. dont le siège à cette époque-là se trouve à Tunis. Cette résistance se répand aussi dans la Vieille ville et les quartiers palestiniens de Jérusalem-Est où ont lieu des actions collectives autour d'enjeux comme la protection de maisons menacées par des colons israéliens qui veulent les occuper, la grève des petits commerces palestiniens aux abords de la Vieille ville ou encore les nombreuses manifestations et confrontations au site du Noble sanctuaire ou Haram Al-Charif (nom arabe pour désigner ce que les Israélien.ne.s appellent le Temple Mount).

17 Cette résistance palestinienne à Jérusalem-Est n'est pas nouvelle. Peu de gens, incluant une bonne partie de chercheur.e.s, savent que, dès 1967, la population locale palestinienne s'est opposée à l'occupation militaire israélienne de Jérusalem-Est, tout comme elle s'est opposée à l'annexion de Jérusalem-Est par le gouvernement israélien en 1981, à l'encontre du droit international. À l'époque, le paradigme dominant est 
celui des luttes de libération nationale. Les analyses en science politique par exemple ne s'intéressaient pas - et s'intéressent encore trop peu - à ces actions autour d'enjeux de la vie quotidienne. Ce récit narratif de la résistance palestinienne à la fois locale et nationale n'avait presque pas été raconté, même en langue arabe.

Jérusalem est une ville au cœur du territoire dorénavant identifié comme israélien, de même que dans les territoires palestiniens occupés par Israël. Ce dernier la considère comme sa "capitale nationale unifiée et indivisible » alors qu'elle est revendiquée par les organisations et la population palestiniennes qui l'ont aussi choisie comme capitale. Dans les discours et documents de l'OLP, Al Quds en arabe, est désignée comme la capitale de l'éventuel État palestinien. Jérusalem a une dimension symbolique forte dans le projet politique palestinien. Cependant, pour l'O.L.P. et les différents partis politiques palestiniens, la lutte était d'abord et avant tout celle de la libération nationale. Conséquemment, elle devait entraîner la fin de l'occupation de l'ensemble des territoires de la Palestine occupée par Israël, incluant Jérusalem. Bref, malgré les discours évoquant l'importance symbolique, religieuse et culturelle de Jérusalem comme capitale du futur État palestinien, le mouvement palestinien n'a pas su développer de stratégie spécifique de résistance pour protéger cette ville pourtant au cœur du territoire à libérer.

19 ALP \& FR : Mais alors, une dernière petite question peut-être avant d'aborder l'expérience de ta thèse : est-ce que ce sujet a été accepté facilement? Travailler sur des mouvements sociaux n'est pas toujours considéré comme légitime dans le champ académique, et à cela s'ajoute la rareté de ce genre d'objet en géographie et études urbaines.

20 AL : On peut dire que j'ai eu un coup de chance. D'une part, j'étais inscrite dans une université qui a vu le jour en 1969 à l'issue de revendications populaires en faveur d'une université publique, laïque, francophone et accessible aux jeunes de milieux populaires où un bon nombre de jeunes professeur.e.s œuvraient dans une perspective critique et souvent engagée. Bien que l'institution ait connu certaines réformes depuis sa fondation, l'UQAM est demeurée et demeure une université où il est possible de travailler sur des questions comme celle-là.

21 D'autre part, Richard Morin, sociologue de formation et professeur au département d'études urbaines de l'UQAM, avait accepté de me diriger sur ce sujet bien qu'il ne le maitrisait pas vraiment. Il connaissait bien la sociologie des mouvements sociaux et avait visité Jérusalem des années auparavant, mais il n'avait jamais travaillé sur le conflit israélo-palestinien. Cependant son ouverture intellectuelle et politique, ses connaissances théoriques et méthodologiques et la confiance qu'il a su me donner, ont joué en faveur de la réalisation d'une telle thèse. Ajoutons que j'avais sollicité deux professeurs palestiniens de l'Université Birzeit, soit le sociologue Salim Tamari et le politologue Saleh Abdel-Jawad, qui avaient accepté d'agir à titre de mentors et que je remercie encore aujourd'hui. J'avais donc mis en place les conditions indispensables pour mener une recherche sur un terrain aussi difficile et miné politiquement.

\section{L'expérience difficile du terrain en Israël-Palestine}

22 ALP \& FR : Comment ça s'est passé sur le terrain? Vu de loin, on imagine que ça devait être difficile voire dangereux. 
23 AL : J'arrive à Ramallah, où j'habiterai de janvier 1994 à novembre 1996, dans le contexte très spécifique du processus de négociations d'Oslo. C'était une période exceptionnelle où on a l'impression de voir l'Histoire (avec un grand $\mathrm{H}$ ) se dérouler devant nous, avec tous les espoirs que ces négociations ont pu susciter de part et d'autre. À peine un mois après mon arrivée, un colon juif israélien d'extrême-droite se rend armé à la mosquée d'Hébron ${ }^{8}$ et tire sur les fidèles faisant 29 morts et 125 blessés palestiniens. Immédiatement après ce massacre, l'information se diffuse partout. De peur de représailles, et aussi pour garder le contrôle sur la population palestinienne des territoires occupés, le gouvernement israélien impose un couvre-feu sur l'ensemble de la Cisjordanie, de la bande de Gaza et de Jérusalem-Est. Personne ne peut sortir de sa demeure, même pour se rendre chez le médecin ou aller à l'école par exemple. Ce jourlà, j'étais en visite chez une copine, une militante palestinienne qui coordonnait une organisation féministe. Apprenant la nouvelle, elle pleurait beaucoup et était extrêmement nerveuse et inquiète des dérapages que cette tragédie pouvait provoquer. À peine un mois après mon arrivée, je connaissais mon premier couvre-feu qui a duré plusieurs jours consécutifs, et ça ne sera pas le seul.

Les données nécessaires à ma recherche se trouvaient surtout à Jérusalem. Mais j'avais choisi de vivre à Ramallah, une ville palestinienne relativement importante située en Cisjordanie, car je voulais connaître, en partie du moins, la vie quotidienne sous occupation militaire et la réalité vécue par la population palestinienne. ${ }^{9}$ Certes, habiter à Jérusalem aurait été plus facile, et m'aurait épargné les déplacements quotidiens entre les deux villes ${ }^{10}$. Dans une certaine mesure, j'aurais eu une plus grande liberté de mouvement, et j'aurais eu accès à davantage d'activités culturelles. Mais je voulais connaître la réalité de l'occupation militaire à laquelle la population palestinienne était soumise depuis 1967, d'où mon choix d'habiter à Ramallah. De cette ville, je me rendais presque tous les jours en taxi collectif à l'Université Birzeit ou à Jérusalem-Est pour ma recherche documentaire, faire des entretiens ou marcher pour mieux connaitre la ville. Très souvent, je travaillais dans un centre de documentation qui se trouvait dans des locaux de la Maison de l'Orient, une immense maison appartenant à la famille Husseini ${ }^{11}$ qui était située dans le quartier Wadi Joz. À l'époque ottomane, c'était un hôtel plutôt luxueux avec un beau jardin. Faisal Husseini, un leader important du parti politique du Fatah et une personnalité publique connue et respectée dans l'ensemble des territoires palestiniens, y avait établi une organisation palestinienne appelée "Arab Studies Society " où se trouvait un centre de documentation et plus tard, un centre de recherche. Là-bas, j'avais accès aux journaux palestiniens, de même qu'à des documents produits par des organisations palestiniennes, et je pouvais échanger avec des chercheur.e.s ou des professionnel.le.s palestinien.ne.s, ce qui me permettait de mieux comprendre leurs points de vue sur les confiscations territoriales par Israël, la construction des colonies de peuplement, etc. Les employé.e.s, les chercheur.e.s, tout le monde était d'une gentillesse et d'une grande générosité envers moi. J'ai pu monter un dossier d'articles de presse tirés des journaux israéliens et palestiniens en remontant jusqu'en 1967, et j'ai réalisé 70 entrevues avec divers acteurs de la ville. ALP \& FR : Par la suite, tu choisis de rentrer à Montréal, tu y retournes par moment?

AL : Oui bien sûr. C'est un "pays » dont je ne me suis pas détachée même si je suis moins active sur le plan de la recherche. Il faut dire que du Québec, la Palestine et Israël sont très loin géographiquement parlant, et les billets d'avion sont chers. Je ne peux donc pas y aller pour travailler une semaine ou dix jours par exemple, ça ne vaut pas 
vraiment le coup si je prends en compte le temps des déplacements. Je « compense » un peu en encadrant des étudiant.e.s. J'ai dirigé deux étudiantes de géographie qui ont réalisé leur mémoire de maîtrise sur la stratégie israélienne d'appropriation du territoire palestinien. De plus, j'encadre un doctorant qui travaille sur les références identitaires mobilisées par des organisations palestiniennes établies dans les camps de réfugiés. Sur une note plus personnelle, à l'été 2017, j’y ai amené mon fils de 17 ans. Je voulais qu'il connaisse cette région qui me tient tant à cœur !

\section{Les références intellectuelles} rédigé un ouvrage majeur sur les structures et l'agentivité en intégrant à son approche le rapport espace-temps (Giddens, 1987). Son ouvrage m'a permis de saisir que la transformation de Jérusalem et plus spécifiquement Jérusalem-Est, n'est pas uniquement le résultat de la stratégie israélienne qui pourtant a le pouvoir, mais bien la confrontation des interventions israéliennes et des actions palestiniennes qui doivent être comprises dans un rapport dialectique.

J'avais lu aussi Milton Santos ${ }^{12}$, le géographe brésilien, Armand Frémont ${ }^{13}$ qui avait développé la notion d'espace vécu, Antoine Bailly ${ }^{14}$ et quelques autres encore. Mais il demeure c'est l'un des ouvrages du géographe français Guy Di Méo ${ }^{15}$ qui m'a le plus influencée. Dans son ouvrage L'homme, la société et l'espace (1991), ce dernier propose un cadre théorique qui repose sur une approche structuraliste à laquelle il intègre des notions empruntées à la géographie humaniste notamment, ce qui permet de se distancier d'une lecture déterministe qui accorderait trop de poids aux structures au détriment des pratiques des agent.e.s et des acteur.trice.s. Son approche permet d'appréhender la transformation des structures, qui combinées les unes aux autres selon différents arrangements, "produisent " les territoires dans un temps long et court à la fois. Ces arrangements qui découlent des pratiques et des stratégies des acteur.e.s, expliquent les spécificités des territoires aux différentes échelles. Cette 
approche théorique qui s'opérationnalise relativement facilement, allait me permettre d'appréhender les principales transformations géographiques, économiques, politiques et idéologiques de Jérusalem qui découlent à la fois des pratiques israéliennes et palestiniennes.

La notion de formation socio-spatiale (FSS) mise de l'avant par Guy Di Méo dans cet ouvrage, a vraiment facilité ma lecture du processus de transformation de Jérusalem, et de Jérusalem-Est. En effet, j'ai pu voir comment les acteur.trice.s, en particulier les dirigeant.e.s israélien.ne.s, ont extrait Jérusalem-Ouest, puis Jérusalem-Est de la formation socio-spatiale arabe (plus spécifiquement jordanienne) dans laquelle elle était située depuis l'époque ottomane pour l'intégrer dans une nouvelle formation socio-spatiale. Les interventions israéliennes ont eu comme effet de déstructurerrestructurer Jérusalem à la fois dans sa composante géographique, mais aussi dans ses composantes économique, idéologique et politique. De plus, bien que Di Méo ne le développait pas assez quant à moi, il a intégré à son schéma conceptuel les pratiques des acteur.trice.s et des agent.e.s dans le processus de construction sociale qui donne lieu aux FSS. Et c'est ainsi que les perspectives de Di Méo et Giddens m'ont permis de comprendre que les composantes structurelles qui "produisent» les territoires sont produites à la fois par les acteur.trice.s et les agent.e.s, et ces dernier.ere.s sont " façonné.e.s " eux-mêmes, elles-mêmes par les structures. Giddens qui parle de structurationnisme (plutôt que de structuralisme), ajoute également une autre considération : les structures sont à la fois contraintes et opportunités. On entend ici qu'elles ne sont ni fixes, ni déterminées et selon le contexte, les lieux, la période temporelle, les acteur.trice.s et agent.e.s ont toujours une "marge de manœuvre » et peuvent envisager le recours aux structure comme opportunités. Bref, je suis partie de la thèse de Di Méo et de celle de Giddens pour développer un cadre théorique que j'ai bonifié en y ajoutant des éléments théoriques empruntés à la sociologie des mouvements sociaux urbains développée par Castells notamment.

ALP \& FR : Est-ce que tu peux nous donner les principaux résultats de ces analyses?

AL: En ayant recours au concept de FSS, j'ai développé un cadre opératoire qui m'a permis d'identifier plus facilement les transformations opérées sur la ville de Jérusalem. J'ai donc été en mesure de démontrer qu'à la suite de la division de la ville de Jérusalem en 1948, chacune des deux parties de la ville a été incorporée dans une formation socio-spatiale différente. En effet, Jérusalem-Ouest a fait partie de la nouvelle formation socio-spatiale que constitue alors Israël, et Jérusalem-Est s'est trouvée insérée dans la FSS de la Jordanie. Puis, après la Guerre de juin 1967 (appelée Guerre des Six jours en Israël), les dirigeants israéliens ont occupé militairement Jérusalem-Est, et ont commencé très rapidement à opérer des transformations urbaines afin qu'elle soit « unifiée » en une seule ville sous le contrôle total israélien. D'ailleurs, l'une des premières mesures adoptées par les leaders israéliens, avant même l'adoption du cessez-le-feu, est de démolir complètement un quartier de la Vieille ville de Jérusalem, le quartier des Maghrébins, situé juste en face du Mur des Lamentations, afin d'élargir la place dédiée aux dévot.e.s. Puis, un général israélien est mandaté pour tracer de nouvelles limites politico-administratives municipales afin d'élargir le territoire municipal. En d'autres mots, on modifie très rapidement la composante spatiale de la ville.

Sur le plan politique, les leaders d'Israël ont créé des "faits" pour imposer leur légitimité politique et faire reconnaître leur pouvoir politique sur la ville. Dans les faits, 
comment cela s'opère-t-il ? En 1967, les dirigeant.e.s israéliens abolissent la municipalité jordanienne, forcent le maire palestinien de l'époque à s'exiler, expulsent les conseillers municipaux palestiniens pour imposer une seule municipalité israélienne pour l'ensemble des habitants juifs israéliens et palestiniens de la ville. En d'autres termes, Israël créé une seule municipalité pour Jérusalem-Ouest et JérusalemEst, prétendant offrir les services municipaux et desservir d'équipements et d'infrastructures les différents quartiers de l'ouest ou l'est de la ville, qu'ils soient juifs ou palestiniens, espérant ainsi imposer leur légitimité politique sur l'ensemble de la ville. En 1981, Israël annexe Jérusalem-Est au sein de l'État israélien et impose ses lois et règlements à la population palestinienne de la ville.

Par ailleurs, afin que Jérusalem devienne de facto la "capitale éternelle de l'État d'Israël », les dirigeant.e.s israélien.ne.s ont tenté de convaincre les pays amis de déménager leur ambassade de Tel Aviv à Jérusalem, essayant ainsi de faire reconnaître sa «légitimité » politique à l'échelle internationale. De plus, les dirigeant.e.s israélien.ne.s ont recours à des symboles pour affirmer l'identité juive israélienne de la ville, voire pour la créer.

De leur côté, dès les premières années, les habitant.e.s palestinien.ne.s de Jérusalem-Est ont refusé de reconnaître cette "légitimité " politique. Pour eux, cela équivalait à reconnaître l'occupation israélienne qui dans les faits, contrevient aux dispositions du droit international.

Sur le plan culturel, comme le démontre bien l'historien israélien Meron Benvenisti dans son ouvrage City of Stone: Hidden History of Jerusalem publié en 1996, l'histoire de la ville présentée au musée $L a$ Tour de David, est celle des conquérant.e.se.s. Le récit narratif à l'entrée du musée repose sur l'historiographie israélienne qui propose une relecture de l'histoire de la ville qui s'inscrit dans la perspective colonialiste de l'État d'Israël. C'est d'ailleurs pour ces mêmes raisons que l'État israélien, peu importe le gouvernement au pouvoir, accorde une aussi grande importance aux fouilles archéologiques à Jérusalem. Les archéologues ont procédé à des fouilles sur le site du Noble Sanctuaire (le Temple Mount pour les Israélien.ne.s) où se trouvent la Mosquée AlAksa et le Dôme du Rocher, quitte à provoquer la colère des Palestinien.ne.s et à contrevenir au droit international.

40 Autre exemple : pour effacer les traces de la présence palestinienne, les noms arabes ou ottomans des rues sont oblitérés et remplacés par des noms juifs qui se réfèrent à l'histoire ancienne ou récente d'Israël. Évidemment, les drapeaux israéliens, un puissant marqueur identitaire, flottent partout, tant dans la Vieille ville que dans la nouvelle ville.

41 Sur le plan économique et contrairement à la majorité des métropoles dans le monde, Jérusalem n'est pas une ville dotée d'une économie forte et diversifiée. Dans son histoire ancienne et encore aujourd'hui, les principales activités économiques sont développées autour des sites religieux, de la Vieille ville, et des infrastructures touristiques. Il y a bien sûr des activités commerciales dans la ville et en périphérie, on retrouve des activités industrielles, mais rien de majeur. En d'autres mots, les principaux enjeux de transformation dans la ville de Jérusalem ne sont pas liés à des enjeux d'ordre économique comme c'est le cas dans d'autres métropoles ou capitales du monde. Ici, force est de constater que ça va à l'encontre d'une lecture marxienne voulant que la structure économique soit déterminante en dernière instance! 

Guy Di Méo dans son schéma conceptuel sur la FSS. À mon avis, le poids démographique d'une population a une influence sur les différentes composantes constitutives de la FSS. Cependant, la grande majorité des chercheur.e.s de sciences sociales ne tiennent pas suffisamment compte de cet élément dans leur analyse des processus de structuration et de transformation des territoires. Dans le conflit israélo-palestinien, si nous saisissons la nature colonialiste et théocratique de l'État-nation israélien, nous devons tenir compte de la composante démographique (ici le poids de la population juive par rapport à la population palestinienne) et l'étudier en relation à la composante idéologique (religion et culture) du projet sioniste. L'État d'Israël a été créé pour rassembler les juif.ve.s du monde entier dans un État juif, d'où la fameuse «loi du retour » qui garantit à tout Juif.ve. le droit d'immigrer dans ce pays. Puisqu'il était et qu'il demeure encore presqu'impossible de procéder à une nouvelle expulsion massive ${ }^{16}$ de la population palestinienne sans fragiliser la position d'Israël vis-à-vis de la communauté internationale, il est impératif que la très grande majorité de la population qui habite dans la capitale de l'État d'Israël soit juive. Dès 1967, sous la recommandation d'un général militaire, il a été décidé que la population totale vivant dans la Jérusalem « unifiée » devait être composée de $72 \%$ de la population juive et de moins de $28 \%$ de non-juif.ve.s. Pour les leaders irsaélien.ne.s, cet objectif est fondamental pour créer et consolider une légitimité juive dans l'ensemble de la ville annexée par Israël en 1981.

Bref, en ayant recours au concept de FSS, je suis en mesure de mettre en lumière les principales politiques et mesures adoptées par les dirigeant.e.s israélien.ne.s des gouvernements local et national, pour transformer les composantes politique, idéologique, et géographique et économique qui structurent Jérusalem, et en particulier Jérusalem-Est.

Pour compléter mon étude de ce processus de déstructuration-restructuration de la FSS de Jérusalem-Est, je me concentre par la suite sur l'étude des pratiques et stratégies palestinienne qui depuis 1967, ont opposé une résistance aux interventions israéliennes pour s'approprier la ville. C'est là que la recherche sur les luttes urbaines et les mouvements sociaux dont j'ai parlé précédemment va me permettre d'appréhender le rôle des mouvements sociaux palestiniens, tant à l'échelle locale que nationale.

\section{Le poste de géographie sociale et la poursuite de recherches « engagées"}

ALP \& FR : Une fois la thèse soutenue, tu as la chance d'obtenir un poste à l'université et ton intérêt pour les mouvements sociaux ne va pas disparaître. Est-ce que tu peux nous dire un petit mot sur ces recherches ultérieures?

AL: Mon intérêt pour les mouvements sociaux, et leur rapport au territoire, va perdurer tout au long de ma carrière. À titre d'exemple, j'ai travaillé sur les pratiques et stratégies d'organisations féministes afin de faire entendre les voix et les besoins des femmes dans la ville par la municipalité. Je me suis aussi penchée sur les actions collectives portées par des femmes et groupes de femmes au Brésil et au Québec afin de mieux faire valoir les besoins spécifiques des femmes en matière d'habitation. Cet intérêt pour les mouvements sociaux va aussi m'amener à travailler directement avec 
des groupes et organisations communautaires dans une perspective du Droit à la ville. En d'autres mots, en tant que chercheure engagée, j'ai pris le pari de travailler non pas sur les mouvements sociaux mais bien avec les mouvements sociaux, ce qui implique de faire de la recherche autrement...

\section{BIBLIOGRAPHY}

BENVENISTI M. (1996), City of Stone: Hidden History of Jerusalem, Berkeley, University of California Press.

CASTELLS M. (1983), The City and the Grassroots: a Cross-Cultural Theory of Urban Social Movements, Berkeley \& Los Angeles, University of California Press, « California Series in Urban Development » no 2.

FRÉMONT A., CHEVALIER J., HÉRIN R., RENARD J. (1984), Géographie sociale, Paris, Masson.

DI MÉO G. (1991), L'Homme, la société et l'Espace, Paris, Anthropos.

DUMPER M. (1997), The Politics of Jerusalem since 1967, New York, Columbia University Press.

FRÉMONT A. (1999) [1976], La région, espace vécu, préface à la deuxième édition, Paris, Flammarion, coll. «Champs ».

GIDDENS A. (1987), La constitution de la société : éléments de la théorie de la structuration, Paris, Presses universitaires de France.

HENTSCH T. (1988), L'Orient imaginaire : la vision politique occidentale de l'Est méditerranéen, Paris, Les Éditions de Minuit.

SANTOS M. (1971), Les villes du Tiers monde, Paris, Génin : Librairies Techniques.

SANTOS M. (1984), Pour une géographie nouvelle. De la critique de la géographie à une géographie critique, Paris, Publisud.

\section{Principales publications d'Anne Latendresse}

MORIN R., PICHÉ C., LATENDRESSE A. (1999), « Développement local et développement régional : quel arrimage ? ", in LAFONTAINE D. et THIVIERGE N. (dir.), Les régions fragiles face à la mondialisation. Stratégies communautaires, technologiques et culturelles d'innovation et de valorisation, Rimouski, GRIDEQ-GRIR.

LATENDRESSE A. (1997-1998), «Israeli Approaches on Jerusaelem », in MOUGHRABI F., AKASH M. (dir.), The Open Veins of Jerusalem. The Arab American Journal of Cultural Exchange, Maryland, Jussoor.

LATENDRESSE A. (1997), « Espace urbain et mobilisation sociale à Jérusalem-Est », in BOCCO R., DESTREMEAU B., HANNOYER J. (dir.), Palestine, Palestiniens. Territoire national, espaces communautaires, Amman, Centre d'études et de recherches sur le Moyen-Orient contemporain. 
LATENDRESSE A. (2005). « Municipal Reform and Public Participation in Montreal's Urban Affairs : Break or Continuity?», in BOOTH PH., JOUVE B. (dir.), Metropolitan Democraties. Transformations of the State and Urban Policy in Canada, France and Great Britain, Hampshire, Ashgate, pp. 117-131.

BEAUDET P., LATENDRESSE A. (2006), « Le Moyen-Orient : rupture ou continuité ? » in KLEIN J-L. et LASSERRE F. (dir.), Le monde dans tous ses États. Une approche géographique, Sainte-Foy, Presses de l'Université du Québec, Coll. « Géographie contemporaine ».

LATENDRESSE A. (2007), La ville, un espace investi par les femmes et les groupes de femmes?, communication présentée dans le cadre du 4 e Congrès de recherches féministes de la francophonie plurielle. Éditions Remue-ménage.

LATENDRESSE A., MASSÉ B. (2008), « L'homo urbanus : enjeux et perspectives de l'urbanisation dans le monde en développement ", in BEAUDET P., HASLAM P. et SCHAFER J. (dir.), Introduction au développement international, Les Presses de l'université d'Ottawa.

LEVY C., CARLE-MARSAN M., LATENDRESSE A. (2013), « Femmes et mouvement populaire d'habitation au Brésil : vers une féminisation des luttes pour le droit au logement? », Économie et Solidarités, vol. 43, no 1-2, pp. 10-38.

DOI : https://doi.org/10.7202/1033273

LEVY C., LATENDRESSE A., CARLE-MARSAN M. (2016), « Gendering the urban social movements and public housing policy in Sao Paulo », Latin American Perspective, vol. 44, no 3, pp. 9-27.

LATENDRESSE A., BORNSTEIN L., REID J. (2017), « Urban Development: Cities in the Global South Cities ", in HASLAM P. A., SCHAFER J. et BEAUDET P. (dir.). Introduction to International Development: Approaches, Actors, Issues and Practices, Don Mills, Oxford University Press.

LATENDRESSE A., CUNHA L. F. (2018), « Praticas insurgentes e planejamento urbano : as operaöes populares de planejamento em Pointe-Saint-Charles Montréal, Qc », Urbe. Revista Brasileira de Gestão Urbana, vol. 11

DOI : https://doi.org/10.1590/2175-3369.011.002.ao15

MARTIN P., A. LATENDRESSE A. (2019), « Féministes tant qu'il le faudra! The Multiple Possibilities of Francophone Feminist Geographies au Québec/Canada », Gender, Place and Culture, vol. 26, no 7-9, pp. 976-987

DOI: 10.1080/0966369X.2018.1552250

BERGERON-GAUVIN J.V., DUFOUR P., LATENDRESSE A. (à paraître), « Une brèche féministe dans les luttes relatives au logement : l'expérience d'Information-Ressources Femmes et Logement, 1986-1995 », Les Cahiers de géographie du Québec.

\section{APPENDIXES}

Chronologie du parcours

1978-1980 Deux années collégiales (pré-universitaires) au CÉGEP Lévis-Lauzon en sciences humaines avec mathématiques. Membre de l'exécutif de l'Association étudiante de 1978 à 1979.

1982-85 Etudes en communication, profil journalistique à l'Université du Québec à Montréal (UQAM). Pendant cette période, elle s'intéresse de façon spécifique au Machrek, notamment à la guerre au Liban et au conflit israélo-palestinien. Au cours de ces trois années, elle est active au sein du Comité de solidarité UQAM-Palestine, et aussi 
du comité Femmes-UQAM. De plus, elle écrit dans le journal étudiant Le Montréal Campus.

En 1984, le Centre d'information et de documentation sur le Mozambique et l'Afrique australe (CIDMAA), une petite ONG établie à Montréal, la sollicite avec d'autres étudiant.e.s pour organiser une campagne de solidarité sur le droit à l'éducation des Sud-Africain.e.

À l'été 1984, année internationale de la jeunesse, elle travaille dans un camp d'été qui rassemble des jeunes des Premières Nations et des jeunes nouveaux arrivant.e.s (immigrant.e.s).

1986-1988 Certificat d'études arabes à l'Université de Montréal.

1987-1988 En 1987, elle obtient une bourse d'études de l'AUPELF (à l'époque Association universitaire de langue partiellement et entièrement de langue française) qui lui permet de suivre un cours intensif de langue arabe à l'Institut des langues vivantes de Bourguiba à Tunis.

La première Intifada (soulèvement populaire) palestinienne éclate en décembre 1987. À Montréal, plusieurs militant.e.s et organisations créent la Coalition pour la paix et la justice en Palestine dont elle devient membre.

À l'été 1988, elle obtient un contrat de travail du Centre d'études arabes pour le développement (CEAD). À l'automne de la même année, à la suite d'un concours, elle obtient le poste de coordonnatrice du CEAD.

Toujours en 1988, alors qu'elle est à l'emploi du Centre d'études arabes pour le développement, elle effectue son 1er séjour dans les territoires palestiniens occupés par Israël. Elle visite des organismes palestiniens qui offrent des services dans le domaine de la santé, de l'agriculture et rencontre des intellectuels et militant.e.s palestinien.ne.s.

1988-90 En soutien à la lutte du peuple palestinien, le CEAD crée un groupe de solidarité afin de mobiliser des jeunes du Québec. Ce groupe est appelé Salam, groupe pour la paix en Palestine. L'un des objectifs de Salam est de sensibiliser les jeunes du Québec aux réalités de l'occupation militaire israélienne et aux violations des droits humains des Palestinien.ne.s en organisant des visites dans les territoires palestiniens. A leur retour, ces jeunes mènent des activités de solidarité avec le peuple palestinien comme des tournées de conférences dans les universités et collèges.

A l'été 1990, Anne Latendresse accompagne un groupe de jeunes du Québec qui effectue un court séjour dans les territoires palestiniens occupés. Un mois avant leur arrivée, elle fait un voyage dans la région avec son copain, lui aussi un militant actif au CEAD. Ce voyage par la voie terrestre la mène d'Istanbul à Jérusalem. Ensemble, ils traversent la Turquie, la Syrie, la Jordanie et traverse en Cisjordanie. Tous leurs déplacements se font par train, autobus et taxis collectifs ce qui lui permet de voir les paysages et de discuter un peu avec les habitant.e.s de ces pays. Ce voyage constitue l'événement déterminant dans son choix de se diriger en géographie. Lors de ce séjour, elle a compris que les enjeux territoriaux étaient au cœur des conflits au Proche-Orient.

1989-1990 À son retour de voyage, elle fait une demande d'admission au programme de Maîtrise de géographie à l'UQAM. Comme elle n'a pas de baccalauréat en géographie, on lui demande de faire cinq cours de propédeutique. En janvier, elle suit ses premiers 
séminaires de géographie en ayant en tête de réaliser une recherche de maîtrise sur le conflit israélo-palestinien.

1991 A l'automne, elle entre au programme de doctorat en études urbaines à l'UQAM. Elle entreprend une recherche sur le processus de transformation de Jérusalem-Est et les pratiques de résistance des Palestinien.ne.s pour protéger le territoire et l'identité de la ville.

Au cours de ces années, elle travaille à titre d'assistante de recherche sur le développement économique communautaire à Montréal dans le cadre d'un projet dirigé par Richard Morin, son directeur de thèse et professeur en études urbaines.

1999 Après le dépôt de sa thèse, elle réalise un contrat de travail pour l'ONG Alternatives qui porte sur l'expérience de budget participatif menée à Porto Alegre au Brésil.

1999-2000 Elle obtient une bourse post-doctorale et est accueillie par le Center for Urban and Community Studies, un centre de recherche affilié à l'Université de Toronto 2000-2001 En septembre 2000, de retour à Montréal, elle enseigne deux cours de Ier cycle au département de géographie de l'UQAM. En février 2001, elle postule au poste de professeur.e en géographie sociale, et l'obtient.

2001 En août, elle débute sa carrière à titre de professeure adjointe de géographie à l'UQAM

2001-2009 En parallèle de ses activités académiques, elle s'implique activement avec le Centre d'écologie urbaine de Montréal et l'organisme Alternatives, et offre des formations sur la démocratie participative et l'écologie. Elle contribue à la création d'une Coalition d'organismes communautaires qui promeut le Droit à la ville, et organise cinq sommets citoyens de Montréal entre 2001 et 2009.

Elle participe aussi à deux éditions du Forum social Québec (2007-2009), cette fois-ci à titre de conférencière et personne-ressource.

2009-2015 Elle est nommée directrice adjointe puis directrice (2010) du Centre d'études et de recherches sur le Brésil (CERB) établi à l'UQAM. Il s'agit d'un centre de recherches et un carrefour d'activités sur le Brésil (colloques étudiants, série de séminaires, accueil de professeur.e.s. et d'étudiant.e.s du Brésil, etc.).

2017-2019 Elle devient directrice des programmes de premier cycle, département de géographie.

\section{NOTES}

1. À la suite d'une réforme municipale, la ville de Lauzon a été fusionnée à d'autres villes pour devenir la ville de Lévis.

2. Au Québec, après avoir terminé leurs études secondaires, les étudiant.e.s agé.e.s de 16 ou 17 ans qui veulent poursuivre leurs études, s'inscrivent au Cegep (Collège d'enseignement général et professionnel). Ils et elles y étudient deux ans s'ils et elles veulent poursuivre à l'université par la suite. Ceux et celles qui veulent acquérir une formation technique y étudient pendant trois ans et obtiennent leur diplôme d'études techniques ou professionnelles. 
3. Nakba est un mot qui signifie "catastrophe » en arabe, et désigne le déplacement forcé de 700000 à 900000 Palestinien.ne.s (selon différentes sources), à la suite de la première guerre israélo-arabe en 1947-1948, et de la création de l'Etat d'Israël en 1948.

4. Thierry Hentsch a été professeur au département de science politique de l'UQAM de 1976 jusqu'à sa mort en 2005. Son œuvre a été couronnée de plusieurs prix. L'ouvrage qui m'a marqué à l'époque est: L'Orient imaginaire (1988). https://www.actualites.uqam.ca/2019/memoirethierry-hentsch

5. L'ANC est l'acronyme pour l'African National Congress, un parti politique créé en 1912 qui mène la lutte contre l'apartheid en Afrique du Sud. Pendant de nombreuses années, ce parti, interdit en Afrique du Sud, est établi à l'extérieur du pays. Ce n'est qu'1991, avec l'autorisation du gouvernement, que l'ANC tient ses premières élections libres et démocratiques dans le pays. Ses militant.e.s et leaders, pour la plupart en exil, rentrent au pays pour prendre part à ces élections. C'est là que le fameux leader Nelson Mandela, après avoir passé 17 ans en prison, est élu président de l'ANC. Trois ans plus tard, lors des élections législatives de 1994, il devient le premier président noir de l'Afrique du Sud.

6. Dans les débats qui suivent la publication de l'ouvrage de Castells, certains auteurs dont Chris Pickvance se questionnent sur l'urbanité en question. Qu'est-ce qu'un mouvement social urbain ? Est-il « urbain » parce qu'il se déroule dans la ville? Ou est-il qualifié d'urbain parce qu'il est lié à des enjeux « spécifiquement urbains »?

7. Intifada, mot arabe qui signifie révolte ou soulèvement.

8. Le massacre d'Hébron de 1994, ou massacre de la mosquée d'Ibrahim, a eu lieu le 25 février 1994. Baruch Goldstein, un colon israélien armé, membre du parti nationalistereligieux Kach et Kahane Chai, s'est rendu à la mosquée d'Hébron alors que les fidèles priaient un vendredi du mois de ramadan.

9. Il va de soi que mon statut d'étrangère provenant d'un pays riche, faisait en sorte que même si j'étais étudiante, les conditions dans lesquelles je vivais étaient nettement meilleures que celles de la population palestinienne. Avec mon passeport canadien, je savais qu'à n'importe quel moment je pouvais rentrer chez moi en toute sécurité. Cependant le fait d'habiter dans une ville sous occupation militaire m'a permis d'être témoin de toute l'oppression vécue par la population palestinienne.

10. A cette époque, le mur de séparation n'était pas construit, mais il y avait un barrage militaire qui séparait Jérusalem de la Cisjordanie. Très souvent, l'armée israélienne fermait ce barrage à titre de représailles, empêchant ainsi les travailleur.euse.s de la Cisjordanie et de Gaza de se rendre à leurs lieux de travail et punissant la population palestinienne.

11. La famille Husseini est l'une des deux grandes familles de notables palestiniens établies à Jérusalem. Certains membres de cette famille ont occupé des postes importants comme celui de maire de la ville ou de Grand Mufti de Jérusalem (la plus haute fonction religieuse en Palestine).

12. Milton Santos est le premier géographe noir brésilien qui obtient un poste à l'université. Né en 1926 dans l'État de Bahia, il s'exile en France durant la période de dictature militaire. Parmi ses nombreux ouvrages traduits en français mentionnons Pour une géographie nouvelle (1986) dans lequel il développe un cadre théorique considéré comme novateur à l'époque, et en 1971, Les villes du Tiers monde.

13. Armand Frémont, décédé en mars 2019, a été un géographe influent dans la francophonie. Il a travaillé notamment sur la région et la notion d'espace vécu (1976). http://geoconfluences.enslyon.fr/actualites/veille/breves/armand-fremont-est-mort

14. Aujourd'hui professeur émérite à l'Université de Genève, Antoine Bailly a reçu de nombreux prix et distinctions. D'origine suisse, il a fait ses études doctorales en France sous la direction de Paul Claval. Il a notamment travaillé sur la notion de " perception de l'espace urbain ». Il publie également de nombreux ouvrages pédagogiques portant sur l'épistémologie ou les concepts de géographie humaine. Parmi les plus importants: Les concepts de la géographie humaine (1984), 
l'Introduction à la géographie humaine (1982), qu'il rédige avec Hubert Béguin et qui est rééditée huit fois, l'Encyclopédie de géographie (1992). https://www.cairn.info/revue-espacegeographique-2012-1-page-95.htm

15. Di Méo est une figure marquante de la géographie sociale dans la francophonie. Pour reprendre les mots de la Société de Géographie, il est une «figure centrale de la géographie contemporaine (2017) ». Professeur Émérite de l'Université Bordeaux Montaigne depuis 2012, il a plus de 160 articles parus dans des revues à comité de lecture et de contributions publiées à l'occasion de colloques nationaux ou internationaux, 16 ouvrages en nom propre et il a pris part à une trentaine d'ouvrages collectifs et d'actes de colloques. Parmi ses nombreuses publications : L'Homme, la société et l'Espace (1991), Les territoires du quotidien (1996), Géographie sociale et territoires (1998), L'espace social, lecture géographie des sociétés (2005), Les murs invisibles (2011), Introduction à la géographie sociale (2014), Le désarroi identitaire (2016). Pour en savoir davantage : Garat, Isabelle, Guy Di Méo, géographe, bâtisseur de théories et laboratoires, https://sms.hypotheses.org/25460

16. Après la $\mathrm{Nakba}$ (note $\mathrm{n}^{\circ} 2$ ), en 1967 , à la suite de la deuxième guerre israélo-arabe qui dure six jours, 250000 autres Palestinien.ne.s se voient forcés de quitter le pays.

\section{AUTHORS}

\section{ANNE LATENDRESSE}

Département de géographie, Université du Québec à Montréal (UQAM)

latendresse.anne[at]uqam.ca

\section{ANNE-LAURE PAILLOUX}

Post-doctorante, Géoarchitecture, Université Bretagne Sud

alpailloux[at]gmail.com

\section{FABRICE RIPOLL}

Maître de Conférences, Lab'urba, Université Paris Est Créteil

fabrice.ripoll[at] $\mathrm{u}-$ pec.fr 